\title{
TESTES IN VITRO E IN VIVO UTILIZADOS NA TRIAGEM TOXICOLÓGICA DE PRODUTOS NATURAIS
}

\section{TESTS IN VITRO AND IN VIVO USED IN THE TOXICOLOGICAL SCREENING OF NATURAL PRODUCTS}

\author{
Bednarczuk, V.O'; Verdam, M.C.S²; Miguel, M.D³; Miguel, O.G \\ ${ }^{1}$ Aluno de Mestrado do Programa de Pós-graduação em Ciências Farmacêuticas. \\ ${ }^{2}$ Aluna de doutorado do Programa de Pós-graduação em Ciências Farmacêuticas. \\ ${ }^{3}$ Professor Adjunto de Farmacotécnica do Curso de Farmácia UFPR. \\ ${ }^{4}$ Professor Adjunto de Fitoquímica do Curso de Farmácia UFPR. \\ *e-mail para correspondência: vinicius.bednarczuk@ hotmail.com
}

REC: 04/2010 AC: 05/2010

\begin{abstract}
RESUMO:
A utilização de plantas pela população tem apresentado um notável aumento. Além disso, o interesse das indústrias e institutos de pesquisa também aumentou. Dessa maneira, se faz necessário a triagem toxicológica das espécies. Estudos toxicológicos in vitro se tornam uma opção na triagem em busca de plantas que possuam efeitos tóxicos, além de diminuir custos, obter respostas rápidas e colaborar com o principio dos 3erres (redução, refinamento e substituição). Neste trabalho testes de toxicidade in vitro e in vivo são discutidos. Estão relacionados testes in vitro e/ou alternativos que visam a identificação preliminar de plantas com potencial efeito tóxico e permitem a redução dos animais utilizados na experimentação.
\end{abstract}

Palavras-Chave: Plantas Medicinais, Toxicologia, Testes In Vitro.

\begin{abstract}
:
The use of plants by the population has shown a remarkable increase. Moreover, the interest of industries and research institutes also increased. Thus, it is necessary to toxicological screening of species. In vitro toxicological studies become an option for screening in search of plants that have toxic effects, and reduce costs, quick answers and collaborate with principle of $3 R$ s (replacement, reduction and refinement). This paper tests for toxicity in vitro and in vivo are discussed. Are related in vitro and / or alternative aimed at the preliminary identification of plants with potential toxic effects and allow the reduction of animals used in experiments.
\end{abstract}

Keywords: Medicinal plants, Toxicology, In Vitro Tests

\section{INTRODUÇÃO}

O homem sempre buscou no reino vegetal a cura ou alívio de suas dores (VEIGA-JUNIOR; PINTO, 2005). Embora a prática seja antiga, as informações permanecem em muitos grupos, onde são acumuladas ao longo dos anos e então repassadas entre as pessoas através da oralidade (AMOROZO, 1996). Apesar do intenso consumo das espécies vegetais nas mais diversas formas de processos extrativos, seja como chás, garrafadas ou emplastros, pouco ainda se sabe sobre a toxicologia das espécies utilizadas (ALONSO, J. 2008). 
Se considerarmos a flora brasileira, grande parte ainda permanece não estudada. Desta forma, seus princípios ativos, suas atividades farmacológicas e os dados toxicológicos permanecem desconhecidos. Tal fato causa extrema preocupação, uma vez que as intoxicações são casos de saúde pública. Segundo dados do SINITOX (Sistema Nacional de Informações Tóxico Farmacológicas) do ano de 2008, no Brasil já foram registrados 85.925 casos de intoxicação por uso indevido de plantas e desses, 441 foram a óbito (SINITOX, 2008).

No uso popular, muitas espécies são utilizadas sem que haja estudos toxicológicos. Segundo o conhecimento popular no qual a planta, por apresentar origem natural, provavelmente não acarreta riscos á saúde, reforça ainda mais a necessidade de extrema preocupação com relação ao seu uso. Algumas espécies já foram pesquisadas e sua toxicidade já é conhecida a saber: Comigo-Ninguém-Pode (Dieffenbachia picta Schott), Bico de Papagaio (Euphorbia pulcherrima Willd), Espirradeira (Nerium oleander L.), entre outras. Diante dessas informações faz-se necessária a implementação dos estudos toxicológicos com as espécies vegetais, garantindo a seguridade no uso das mesmas.

Neste contexto, a preocupação com o uso indevido de animais na experimentação sempre foi um assunto amplamente discutido, diversas leis e projetos foram elaborados para que o número de animais na experimentação fosse reduzido e que esses animais possuissem uma boa qualidade de vida antes e durante os experimentos. Hoje no Brasil existem diversos orgãos que controlam o uso de animais em experimentos, como exemplo os comitês de ética responsáveis pela avaliação e aprovação de projetos de pesquisa com animais. A partir do decreto $n \quad 6899$ de 15 de julho de 2009 que dispõe sobre a composição do Conselho Nacional de Controle de Experimentação Animal - CONCEA, estabelecendo normas para o seu funcionamento e de sua Secretaria-Executiva, criou-se o Cadastro das Instituições de Uso Científico de Animais - CIUCA, mediante a regulamentação da Lei n 11.794, de 8 de outubro de 2008, que dispõe sobre procedimentos para o uso científico de animais, e dá outras providências (BRASIL. 2009).

Assim, as leis, discussões, projetos e comitês visam a diminuição do número de animais usados em experimentos toxicológicos, forçam uma reflexão acerca do tema e a implementação de testes preliminares que permitam realizar inferências que possibilitem a redução do uso de animais e uma discussão ampla dos resultados in vitro e in vivo.

O presente trabalho revisa testes preliminares de avaliação da toxicidade para triagem toxicológica de extratos e substâncias de origem vegetal discutindo os princípios dos 3 Rs (redução, refinamento e substituição) da experimentação animal.

\section{ENSAIOS TOXICOLÓGICOS}

Ensaios de atividade biológica são de fundamental importância na triagem de estudo de um vegetal. Os ensaios toxicológicos complementam os ensaios biológicos (MACIEL; PINTO; VEIGA-JUNIOR, 2002) podendo ser abordado sob dois pontos de vista, o primeiro sob ponto de vista comercial onde a Portaria no 116/1996 da Secretaria de Vigilância Sanitária do Ministério da Saúde do Brasil (BRASIL, 1996) que normatiza estudos de toxicidade crônica e aguda para produtos fitoterápicos e o se- 
gundo sob o desenvolvimento tecnológico tornando-se indispensável para validação de processos tecnológicos. (SONAGLIO, D. et al. 2007)

\subsection{Métodos Alternativos e Testes in vitro}

Métodos alternativos e testes in vitro são aqueles métodos ou técnicas que podem substituir ou diminuir o uso de animais na experimentação. Esses testes identificam bem o principio dos 3Rs que são a substituição (replacement)- experimentos que utilizam animais por outro que não necessite, a redução (reduction) redução do número de animais e o refinamento (refinement) com a diminuição da severidade dos processos (REPETTO, G.; REPETTO, M. 1995).

Essas técnicas compreendem a utilização de organismos como: bactérias, fungos, algas e crustáceos além de frações subcelulares presentes no sistema biológico como suspensões celulares, cultivo de tecidos, cultivos celulares, enzimas e proteínas. A finalidade desses ensaios é de substituir os ensaios com animais, ou de poderem servir como prévia de um estudo toxicológico in vivo, complementando e melhorando a sensibilidade e especificidade de estudos com animais (FRAZIER, J.M. 1992).

\subsubsection{Artemia salina}

Um dos métodos alternativos amplamente utilizados em triagem de substâncias de origem vegetal é o método que utiliza o microcustáceo Artemia salina.

Artemia Salina é um microcustáceo da ordem Anostraca utilizado na alimentação de peixes e camarões por seu alto valor nutritivo. Dependendo da alimentação ingerida esse microcrustáceo pode adquirir diversas colorações, suas características é de nadarem com a parte ventral do corpo voltada para cima, ou seja, para a luz e de estarem sempre em locomoção, por serem animais filtradores e dependem disso para alimentar-se e respirar (ASEM, A. 2008).

A utilização da $A$. Salina em estudos toxicológicos preliminares deve-se a simplicidade com que pode ser manuseado. A rapidez e o baixo custo favorecem a utilização em diversos estudos. Ensaios de letalidade são muito utilizados em análises preliminares de toxicidade geral podendo estimar a concentração média letal (CL50) (LUNA, J.S et al., 2005).

Segundo MEYER, et al. (1982) foi estabelecido uma relação entre o grau de toxicidade e a dose letal média, CL50, de extratos de plantas sobre microcrustáceos Artemia Salina, considerando que quando verificados valores acima de $1000 \mu \mathrm{g} / \mathrm{mL}$ e não havendo morte acima de $50 \%$, estes, são considerados atóxicos. Diversos trabalhos vêm tentando correlacionar a toxicidade sobre Artemia Salina com atividades antifúngica, viruscida, antimicrobiana, tripanossomicida e parasiticida.

\subsubsection{Atividade hemolítica}

A hemólise é caracterizada por ruptura do eritrócito com liberação de hemoglobina. A hemoglobina livre no plasma é prejudicial a saúde causando sérios danos em órgãos vitais tais como fígado, rins e coração, sendo necessário dessa maneira a 
observação da referida atividade (CARVALHO et al., 2007). Nesse sentido na triagem de atividades biológicas e toxicológicas de extratos vegetais, faz-se necessária a verificação da atividade hemolítica das espécies estudadas.

Muitas são as metodologias disponíveis para a verificação da atividade hemolítica de espécies vegetais. A organização mundial de saúde - OMS em seu manual de 1998 preconiza a avaliação qualitativa por observação de coloração vermelha decorrente da liberação de hemoglobina (OMS, 1998). Outra alternativa é o método de observação de formação de halos de hemólise em placas de ágar sangue (EFING, 2008). Uma técnica mais recente e recomendada pela Agência Nacional de Vigilância Sanitária - ANVISA recomenda a observação de absorbância em ultra-violeta, sendo este um teste exigido no Guia para avaliação de segurança de produtos cosméticos (ANVISA, 2003). Independente da metodologia escolhida faz-se necessário a avaliação dessa atividade, indicando possíveis danos da espécie estudada sobre os eritrócitos.

\subsubsection{Cultura de células}

Um modelo de avaliação de toxicidade que vem ganhando força entre os pesquisadores é a citotoxicidade. Tal modelo utiliza-se de células de mamífero e avalia os danos causados a célula, a formação ou não de colônias celulares e a viabilidade celular. O uso de culturas de células tem se tornado um modelo muito apreciado, pois são reprodutíveis, sensíveis e rápidos (ROGERO et al., 2003).

Alguns modelos estão amplamente difundidos entre os modelos de citotoxicidade utilizando culturas de células. Entre eles estão a difusão em ágar e o método do corante vermelho neutro.

O modelo de citotoxicidade pelo método de difusão em ágar é uma técnica utilizando culturas celulares semeadas em placas de petri. No controle positivo é utilizado material que possui ação citotóxica como fragmentos de látex, e como controle negativo materiais comprovadamente atóxicos. As placas são analisadas macroscopicamente verificando a presença de halos. Caso positivo, estes são analisados microscopicamente analisando a integridade celular, lise e morte celular. A ocorrência do halo deve-se a liberação do corante vermelho neutro incorporado nas células (ROGERO et al., 2003).

O corante vermelho neutro ou vermelho toluileno é um corante utilizado na histologia para corar células penetrando no interior do núcleo, esse corante também atinge células vivas por isso é utilizado em culturas celulares, sua coloração é vermeIha mas com pH acima de 8 adquiri coloração amarelada (INSTITUTE FOR IN VITRO SCIENCES).

Outro modelo é o de citotoxicidade pelo método de incorporação do vermelho neutro onde extratos das amostras são diluídos com o meio de cultura utilizado na manutenção da linhagem celular. O controle positivo é utilizado para demonstrar a toxicidade da amostra e o controle negativo para demonstrar na prática a resposta celular. O resultado é analisado em espectrofotômetro a $540 \mathrm{~nm}$. A partir dos resultados calcula-se a média da porcentagem de viabilidade celular em relação ao controle de células (100\%), em função da concentração do extrato. Os resultados geram gráfico do qual se obtém a curva de viabilidade celular. Através dessa curva encontra-se o 
índice de citotoxicidade (IC50\%). Com esses dados verifica-se a concentração do extrato que induz $50 \%$ de lise ou morte celular, como consequência a inibição da incorporação do vermelho neutro (CRUZ, A.S. 2003).

\section{TESTES IN VIVO}

Alguns modelos de estudos toxicológicos in vivo abordam ensaios de toxicidade aguda, toxicidade subcrônica, toxicidade crônica, onde este deve abordar mutagenicidade, embriofetotoxicidade, alterações de fertilidade, carcinogenicidade, indução de dependência. (VILAS BOAS, O. M. G. C. 2006)

$\mathrm{Na}$ toxicidade aguda, são utilizados grupos de animais com diferentes doses chegando a $2000 \mathrm{mg} / \mathrm{Kg}$. A dose é administrada uma única vez, e o animal é observado por um período de 15 dias. Posteriormente é verificado se houve morte. Os órgãos são avaliados conforme o peso e fisionomia. Esse teste verifica se uma dose alta do produto testado têm a capacidade de gerar efeitos tóxicos no individuo. (VALADARES, M.C. 2006)

Nos testes de toxicidade sub-crônica e crônica o período de exposição é maior chegando a 6 meses na toxicidade crônica e 3 meses no sub-crônica, utilizando grupos com diferentes doses administradas 3 vezes ao dia. Após o período dos testes os animais são avaliados. Ao contrário da toxicidade aguda onde o objetivo é verificar se uma dose alta causa efeitos tóxicos a toxicidade sub-crônica e crônica tem como objetivo observar se por um longo período de tempo o produto testado causa efeitos tóxicos (VILAS BOAS, O. M. G. C. 2006).

Testes de toxicidade in vivo sempre foram intensamente criticados por grupos de proteção animal. As reflexões feitas a partir do tema fizeram com que o número de animais utilizados nessas metodologias fosse reduzido. Testes como o de toxicidade aguda onde antes eram utilizados de 20 a 30 animais por grupo com doses chegando a $5000 \mathrm{mg} / \mathrm{Kg}$ foram reduzidos para grupos de no máximo 10 animais normalmente utilizando 3 grupos com 5 animais com dose máxima de 2000 mg/Kg. (VALADARES, M.C. 2006).

Diversos fatores influenciam na toxicidade como fatores biológicos: idade, peso corpóreo, temperatura, fatores genéticos, estados nutricionais e patológicos, e outros como: concentração da amostra testada, estado de dispersão (forma e tamanho das partículas), solubilidade nos fluídos orgânicos, afinidade e sensibilidade ao tecido ou organismo humano e fatores da própria substância (CAZARIN, C. C. K. et al. 2004). Diante de tantos fatores que podem influenciar na toxicidade de uma substância os testes in vivo ainda não podem ser substituídos completamente. No entanto, as metodologias podem sofrer redução com a atribuição de testes in vitro na triagem, respeitando os $3 \mathrm{Rs}$ com algumas propostas expostas na tabela 1: 
TABELA 1: AÇÕES E METAS FUNDAMENTAIS PARA O CUMPRIMENTO DOS OBJETIVOS DO PROGRAMA 3 RS

\begin{tabular}{|c|c|c|}
\hline REDUÇÃO & REFINAMENTO & SUBSTITUIÇÃO \\
\hline $\begin{array}{l}\text { - Desenvolver novos protocolos } \\
\text { com a utilização de menor } \\
\text { número de animais por } \\
\text { experimento; } \\
\text { - Evitar a replicação dos estudos } \\
\text { conduzidos in vivo; } \\
\text {-Priorizar estudos com } \\
\text { relevância e que possam ser } \\
\text { extrapolados para espécie } \\
\text { humana. } \\
\text {-Desenvolver novas } \\
\text { metodologias e modelos de } \\
\text { triagem; } \\
\text { - Aperfeiçoar a qualidade técnica } \\
\text { dos ensaios; } \\
\text { - Diminuir o número de animais } \\
\text { em testes retirando do ensaio o } \\
\text { maior número de informações } \\
\text { possíveis. }\end{array}$ & $\begin{array}{c}\text { - Valorizar os avanços científicos } \\
\text { empregando as novas } \\
\text { metodologias. Preocupar-se } \\
\text { com o bem estar dos animais, } \\
\text { reduzindo o sofrimento dos } \\
\text { mesmos. }\end{array}$ & $\begin{array}{l}\text { - Desenvolver novas } \\
\text { metodologias que possam } \\
\text { substituir os ensaios in } \\
\text { vivo. Métodos in vitro e } \\
\text { alternativos, assim como } \\
\text { aqueles que se utilizam de } \\
\text { células humanas devem ser } \\
\text { priorizados. }\end{array}$ \\
\hline
\end{tabular}

Fonte: CAZARIN, C. C. K. et al. 2004

\section{CONCLUSÃO}

A busca por substâncias que possam servir como ponto de partida para o desenvolvimento de novos fármacos tem aumentando o interesse da indústria farmacêutica e das instituições de pesquisa pelos produtos naturais, uma vez que o reino vegetal tem como fornecer estruturas químicas incomparáveis em número e complexidade. Faz-se necessário então, garantir a seguridade de tais moléculas, além de fornecer à sociedade informações quanto ao uso de extratos vegetais. Nesse sentido, os testes preliminares permitem fazer inferências acerca da toxicidade de tais produtos e permitem ainda que se pense em outros testes que avaliem a toxicidade de forma mais ampla. Os testes preliminares funcionam como uma ótima ferramenta nos estudos com plantas medicinais e devem ser implementados, pois auxiliam na diminuição do uso de animais na experimentação o que vem sendo uma preocupação dos Comitês de ética em experimentação animal. Os testes com animais devem ser feitos com ética e respeito ao animal e fornecem informações importantes em relação ao metabolismo e aos efeitos sobre órgãos específicos. Conjuntamente os ensaios fornecem informações fundamentais nas pesquisas com plantas medicinais e permitem que se evite os graves problemas decorrentes do desconhecimento das espécies utilizadas. 


\section{REFERÊNCIAS}

AGÊNCIA NACIONAL DE VIGILÂNCIA SANITÁRIO (ANVISA). Guia para avaliação de segurança de produtos cosméticos. Brasília. 2003

ALONSO, J. Fitomedicina: curso para profissionais da área da saúde. Editora Pharmabooks. São Paulo. 2008. p. 28 - 33.

ASEM, A. Historical record on brine shrimp Artemia more than one thousand years ago from Urmia Lake, Iran. Journal of Biological Research-Thessaloniki., 2008. p. 113 $-114$.

AMOROZO, Maria Christina de Mello. A abordagem etnobotânica na pesquisa de plantas medicinais. In: Di STASI, Luiz Cláudio. Plantas medicinais arte e ciência. Um guia de estudo interdisciplinar. São Paulo: Editora da Universidade Estadual Paulista, 1996. p.87.

BRASIL. Ministério da Saúde. Secretaria de Vigilância Sanitária. Portaria n 116, de 8.8.1996. Diário Oficial da República Federativa do Brasil, 12 ago 1996.

BRASIL. Ministério da Saúde. Secretaria de Vigilância Sanitária. Decreto 6899/09. Diário Oficial da República Federativa do Brasil, 15 de julho de 2009.

CARVALHO et al. Efeito da bomba de infusão de soluções sobre o graus de hemólise em concentrados de hemácias. Revista Brasileira de Hematologia e Hemoterapia. V.29, n. 2. 149-152, 2007.

CAZARIN, C. C. K. et al. Redução, refinamento e substituição do uso de animais em estudos toxicológicos: uma abordagem atual. Revista Brasileira de Ciências Farmacêuticas. vol.40 no.3 São Paulo Julho/Setembro. 2004.

CRUZ, A.S. Teste de Citotoxicidade In Vitro como Alternativa ao Teste In Vivo de Draize na Avaliação de Produtos Cosméticos. Tese de doutorado apresentada no ano de 2003. São Paulo. USP. p. 37 -38.

CRUZ, A.S.; Cuppoloni, K.M.; Martinez, C.H.O.; Gomes, L.F.S. Revista - Instituto Adolfo Lutz, v. 47, n. 1/2, p. 51-57, 1987.

EFING, 2008. Compostos bioativos do material resinoso, subproduto do processamento da erva-mate. Ilex paraguaiensis A. St-Hill. Dissertaçao de mestrado Curso de pos graduação em tecnologia de alimentos. Universidade Federal do Paraná. 108p. 2008.

FRAZIER, J. M. In vitro Toxicity testing. Applications to safety evaluation. New York, Marcel Dekker, Inc., 1992. p. 300.

INSTITUTE FOR IN VITRO SCIENCES. Neutral Red Release Bioassay. http://www. 
iivs.org/pages/methods.php . Acessado em 2 de novembro de 2010.

LUNA, J.S. et al. A study of the larvicidal and molluscicidal activities of some medicinal plants from northeast Brazil. Journal of Ethnopharmacology. 2005. p. 199 - 206.

MACIEL, Maria Aparecida M.; PINTO, Ângelo C.; VEIGA JR, Valdir Veiga; GRYNBERG, Noema F; ECHVARRIA, Áurea. Plantas medicinais: A necessidade de estudos multidisciplinares. Química Nova. v.25, n.3, p.429-438, 2002.

MEYER, B. N., et al. Brine Shrimp: a convenient general bioassay for active plant constituints. Planta Médica, v.45, p.31-34, 1982.

ORGANIZAÇÃO MUNDIAL DA SAÚDE - OMS. Quality control methods for medicinal plants methods. 1998. p. $41-43$.

REPETTO, G.; REPETTO, M. Toxicologia Avanzada. Capítulo 2: Métodos Alternativos: estudios toxicológicos in vitro. Madrid: Ediciones Días de Santos. 1995. p. 37 - 45.

ROGERO et al. Teste in vitro de citotoxicidade: Estudo comparativo entre duas metodologias. Materials research. v.6, n.3, 317-320. 2003.

SINITOX. Sistema Nacional de Informações Tóxico Farmacológicas. http://www.fiocruz.br/sinitox_novo/media/tab02_brasil_2008.pdf Site acessado dia 31 de outubro de 2010.

SONAGLIO, D. et al. Farmacognosia - da planta ao medicamento. Capítulo 13: Desenvolvimento Tecnológico e Produção de Fitoterápicos. 6. ed. Editora da UFRGS; Editora da UFSC. 2007. p. $290-291$.

VALADARES, M.C. AVALIAÇÃO DE TOXICIDADE AGUDA: ESTRATÉGIAS APÓS A "ERA DO TESTE DL50". Revista Eletrônica de Farmácia Vol 3(2)., 2006. p. 93-98.

VEIGA-JUNIOR, V. F. V.; PINTO, A. C., Plantas medicinais: Cura segura? Química nova. v.28, n.3, p. 519-528, 2005. 